Article

\title{
Genetic Polymorphisms Associated with the Neutrophil-Lymphocyte Ratio and Their Clinical Implications for Metabolic Risk Factors
}

\author{
Boram Park ${ }^{1}$, Eun Kyung Choe ${ }^{2, *(\mathbb{D})}$, Hae Yeon Kang ${ }^{3}{ }^{(\mathbb{D}}$, Eunsoon Shin ${ }^{4}$, Sangwoo Lee ${ }^{5}$ \\ and Sungho Won ${ }^{1,6}$ \\ 1 Department of Public Health Sciences, Seoul National University, Seoul 08826, Korea; \\ 90may11@gmail.com (B.P.); sunghow@gmail.com (S.W.) \\ 2 Department of Surgery, Seoul National University Hospital Healthcare System Gangnam Center, \\ Seoul 06236, Korea \\ 3 Department of Internal Medicine, Seoul National University Hospital Healthcare System Gangnam Center, \\ Seoul 06236, Korea; joybell77@hanmail.net \\ 4 DNALink, Inc., Seoul 03759, Korea; esshin@dnalink.com \\ 5 Samsung Electronics, Inc., Suwon 16677, Korea; lsw00kor@gmail.com \\ 6 Institute of Health and Environment, Seoul National University, Seoul 08826, Korea \\ * Correspondence: snuhcr@naver.com; Tel.: +82-2-2112-5742; Fax: +82-2-2112-5635
}

Received: 11 July 2018; Accepted: 6 August 2018; Published: 8 August 2018

\begin{abstract}
Background: The neutrophil-lymphocyte ratio (NLR) is a valuable prognostic or predictive biomarker in various diseases, but the genetic factors that underlie the NLR have not been studied. We attempted to investigate polymorphisms related to NLR phenotype and analyze their ability to predict metabolic risks. Methods: A genome-wide association study was performed with log-transformed NLR using an Affymetrix Axiom ${ }^{\mathrm{TM}}$ KORV1.1-96 Array. Regression models for metabolic risk status were designed using the identified significant single-nucleotide polymorphisms (SNPs). Results: We identified four SNPs near the TMEM116, NAA25, and PTPN11 genes that were associated with the NLR. The top SNP associated with the log-transformed NLR was rs76181728 in TMEM116. A case-control study was performed to analyze the metabolic risks associated with each SNP after adjusting for age, sex, and body mass index (BMI). Three SNPs displayed significant odds ratios (ORs) for increased blood pressure and increased waist circumference. In the regression model for metabolic syndrome, rs76181728 showed a significant association $(\mathrm{OR}=1.465$, $95 \%$ confidence interval $(C I)=1.091-1.969, P=0.011)$ after adjustment for the NLR phenotype. Conclusions: We identified four novel SNPs that are associated with the NLR in healthy Koreans. SNPs in relevant genes might therefore serve as biomarkers for metabolic risks.
\end{abstract}

Keywords: neutrophil; lymphocyte; metabolic risks; polymorphism

\section{Introduction}

Inflammatory status plays an important role in determining the pathophysiology and prognosis of various diseases. Recently, the neutrophil-lymphocyte ratio (NLR) has been explored as a biomarker of inflammation [1]. The NLR has previously been reported as a prognostic or predictive biomarker in a variety of cancers [2-4], metabolic diseases [5], and cardiovascular diseases [6-8]. In a meta-analysis of renal cell carcinoma, a higher NLR predicted poorer overall survival (hazard ratio, 1.82) and poorer recurrence-free/progression-free survival (hazard ratio, 2.18) [3]. In a study performed in an Asian Indian population, the NLR was higher in metabolic syndrome, and as the number of metabolic abnormalities decreased, the NLR also decreased in a linear manner [5]. Finally, in a cohort 
representative of the general population in the United States, the NLR was found to be a predictor of cardiovascular mortality and was used to accurately reclassify patients at intermediate risk of cardiovascular disease according to their Framingham risk score [8].

The NLR represents the balance between leukocytes and neutrophils, and expresses how dominant neutrophils are over lymphocytes. Modulation of the NLR may therefore reflect a deeper imbalance in the immunological responses [1]. This notion is supported by the observation that the NLR is associated with the propagation of various cytokines [1]. However, the genetic mechanisms underlying how the NLR is modulated have not been studied. We propose that NLR-associated genetic factors could affect a variety of diseases before an NLR phenotype manifests. In this study, we evaluated two outcome measures. First, we performed a genome-wide association study (GWAS) to identify genetic polymorphisms that are related to the NLR phenotype. Second, using the results obtained from the GWAS of the NLR phenotype, we investigated the clinical implications of the identified single-nucleotide polymorphisms (SNPs). We chose metabolic syndrome as a representative condition in this analysis. Metabolic syndrome is a disease that has been reported to be related to the NLR level and has a large influence on healthcare. This condition is estimated to affect one quarter of the world's adult population, and $28.8 \%$ of general population have it [1]. We sought to determine whether the polymorphisms identified in this study can be used to predict metabolic syndrome or metabolic risk factors in addition to the impact of NLR phenotype.

\section{Methods}

\subsection{Study Subjects}

We retrospectively used clinical and genetic data stored between 2014 and 2015 in the Gene-Environmental Interaction and Phenotype (GENIE) database for Koreans. The details of the characteristics and structure of the GENIE study are described elsewhere [9]. Briefly, DNA samples were collected from peripheral blood obtained from individuals who participated in comprehensive health checkups. SNP genotyping was performed using an Affymetrix Axiom ${ }^{\mathrm{TM}}$ KORV1.1-96 Array (Thermo Fisher Scientific, Santa Clara, CA, USA) by DNA Link, Inc. From this database, individuals for whom blood neutrophil and lymphocyte counts were available in their health checkup data were included. Individuals with a history of malignancy, acute inflammatory conditions, or infectious diseases were excluded.

\subsection{Clinical Assessment and Definitions}

All blood samples were obtained after the subjects had fasted for at least $10 \mathrm{~h}$. To diagnose metabolic syndrome, we followed the criteria suggested by the American Heart Association [10], which requires at least three of the following metabolic risks: increased waist circumference (males $>102 \mathrm{~cm}$; females $>88 \mathrm{~cm}$ ); elevated triglycerides $(\geq 150 \mathrm{mg} / \mathrm{dL})$; reduced high-density lipoprotein (HDL) cholesterol (males $<40 \mathrm{mg} / \mathrm{dL}$; females $<50 \mathrm{mg} / \mathrm{dL}$ ); elevated fasting glucose (fasting glucose $\geq 100 \mathrm{mg} / \mathrm{dL}$ or the use of medications for hyperglycemia); and elevated blood pressure ( $\geq 130 / 85 \mathrm{mmHg}$ or the use of medications for hypertension). The consumption of more than $140 \mathrm{~g}$ of alcohol per week was regarded as a yes in the questionnaire. The subjects were grouped as non-smokers/ex-smokers vs. current smokers.

\subsection{Ethics Statement}

The Institutional Review Board of the Seoul National University Hospital approved this study protocol (H-1512-055-727) and waived the need for informed consent. The study was performed in accordance with the Declaration of Helsinki. 


\subsection{Genotyping, Quality Control and Imputation}

We performed systematic quality control in the procedure described below as presented elsewhere [11]. Samples matching the following criteria were excluded: sex inconsistencies; call rate up to $97 \%$; and related- or cryptically-related individuals (identical by state (IBS) $>90 \%$ ). SNPs were filtered out if the minor allele frequency was approximately $5 \%$, the excessive missing genotype was more than $3 \%$, or there was a significant deviation from the Hardy-Weinberg equilibrium permutation test $\left(P<10^{-5}\right)$. We also excluded SNPs that were likely to indicate false positive associations due to incorrect clustering. After quality control was performed, 345,072 SNPs remained.

GWAS imputation was carried out using SHAPEIT2 v2.r837 [12] and IMPUTE2 [13] version 2.3.2 for pre-phasing the data and genotype imputations. We used 1000 Genomes Phase 3 haplotypes as the reference panel. The imputation accuracies of the analyzed SNPs were confirmed with an info metric obtained from IMPUTE2, and SNPs less than 0.5 INFO were removed. After imputation, a total of $3,693,205$ SNPs from 7303 individuals were used for the analysis. A population stratification analysis was performed using principal component analysis (Figure S1) to assess the influence of race using the EIGENSIFT package version 6.1.4.

\subsection{Statistical Analysis}

The NLRs were log-transformed to approximate a normal distribution. We categorized the NLR into normal NLRs ( $<$ mean NLR +1 standard deviation) vs. increased NLR ( $\geq$ mean NLR +1 standard deviation) based on an arbitrary cut-off value (mean NLR +1 standard deviation). The calculated cut-off value was 2.84. We used multiple regression models to determine the associations between the log-transformed NLRs and the SNPs in the PLINK software packages, version 1.9 [14]. Principal component scores were estimated with WISARD software (http:/ / statgen. snu.ac.kr/wisard/). Age, sex, and body mass index (BMI) were used as covariates in the additive model. The results were verified using discovery and validation sets. Subjects enrolled between January 2014 and April 2015 were included in the discovery set $(n=4986)$, and subjects enrolled between May 2015 and December 2015 were included in the validation set $(n=2271)$. SNPs that fulfilled the Bonferroni corrected $P$-value, $5 \times 10^{-8}$, in the discovery set were re-evaluated in the validation set. $P$-values less than 0.05 were considered statistically significant in the validation set.

We also analyzed the associations of log-transformed white blood cell (WBC) counts, neutrophil counts, and lymphocyte counts with SNPs because the NLR is related to these parameters.

Using a multiple logistic regression analysis, we performed a case-control study for each metabolic risk factor according to the significant SNPs in additive models after adjusting for age, sex, and BMI. Odds ratios (ORs), 95\% confidence intervals (CIs), and corresponding $P$-values were calculated for candidate SNPs.

Finally, from the discovery data, we designed various regression models for metabolic risk evaluation. We used the most significant SNP according to dominant genotypic modeling in the multiple logistic regression analysis after adjusting for various factors, such as age, sex and BMI in model 1; age, sex, smoking status, alcohol consumption, and BMI for model 2; age, sex, BMI, and log-transformed NLR for model 3; and age, sex, smoking status, alcohol consumption, BMI, and log-transformed NLR for model 4.

The R statistical software (Version 3.4.4) package was used for statistical analyses, and $P$-values less than 0.05 were considered significant.

\section{Results}

\subsection{Characteristics of the Study Population}

The characteristics of the subjects in the discovery and validation sets are described in Table 1. 
Table 1. Baseline characteristics of the study population.

\begin{tabular}{|c|c|c|c|c|}
\hline & Total & Discovery Set & Validation Set & \\
\hline$N$ & $(N=7257)$ & $(N=4986)$ & $(N=2271)$ & $P$ \\
\hline Age & $50.5 \pm 10.2$ & $50.6 \pm 10.4$ & $50.2 \pm 9.7$ & 0.081 \\
\hline NLR & $1.9 \pm 0.9$ & $1.9 \pm 0.9$ & $1.9 \pm 0.9$ & 0.075 \\
\hline *NLR & & & & 0.230 \\
\hline Normal & $6657(91.7 \%)$ & $4487(90.0 \%)$ & $2022(89.0 \%)$ & \\
\hline Increased & $600(8.3 \%)$ & $499(10.0 \%)$ & $249(11.0 \%)$ & \\
\hline WBC count $\left(\times 10^{3} / \mu \mathrm{L}\right)$ & $5.4 \pm 1.5$ & $5.3 \pm 1.5$ & $5.5 \pm 1.5$ & 0.001 \\
\hline Neutrophil count $\left(\times 10^{3} / \mu \mathrm{L}\right)$ & $3.1 \pm 1.2$ & $3.1 \pm 1.2$ & $3.2 \pm 1.2$ & 0.001 \\
\hline Lymphocyte count $\left(\times 10^{3} / \mu \mathrm{L}\right)$ & $1.7 \pm 0.5$ & $1.7 \pm 0.5$ & $1.7 \pm 0.5$ & 0.018 \\
\hline High sensitivity C-reactive protein & $0.1 \pm 0.3$ & $0.1 \pm 0.3$ & $0.1 \pm 0.4$ & 0.127 \\
\hline Gender & & & & 0.007 \\
\hline Men & $4208(58.0 \%)$ & $2838(56.9 \%)$ & $1370(60.3 \%)$ & \\
\hline Women & $3049(42.0 \%)$ & $2148(43.1 \%)$ & $901(39.7 \%)$ & \\
\hline BMI & $23.1 \pm 3.0$ & $23.1 \pm 3.0$ & $23.3 \pm 3.0$ & 0.009 \\
\hline Smoking & & & & 0.985 \\
\hline None or ex-smoker & $3196(50.4 \%)$ & $2184(50.5 \%)$ & $1012(50.3 \%)$ & \\
\hline Current smoker & $1997(31.5 \%)$ & $1363(31.5 \%)$ & $634(31.5 \%)$ & \\
\hline Missing & $1149(18.1 \%)$ & $782(18.1 \%)$ & $367(18.2 \%)$ & \\
\hline Alcohol consumption & & & & 0.026 \\
\hline No & $4583(63.2 \%)$ & $3161(63.4 \%)$ & $1422(62.6 \%)$ & \\
\hline Yes & $1559(21.5 \%)$ & $1033(20.7 \%)$ & $526(23.2 \%)$ & \\
\hline Missing & $1115(15.4 \%)$ & $792(15.9 \%)$ & $323(14.2 \%)$ & \\
\hline Diabetes medication & & & & 0.482 \\
\hline No & $6896(95.2 \%)$ & $4744(95.3 \%)$ & $2152(94.9 \%)$ & \\
\hline Yes & $350(4.8 \%)$ & $234(4.7 \%)$ & $116(5.1 \%)$ & \\
\hline Hypertension medication & & & & 0.640 \\
\hline No & $6030(83.2 \%)$ & $4150(83.4 \%)$ & $1880(82.9 \%)$ & \\
\hline Yes & $1216(16.8 \%)$ & $828(16.6 \%)$ & $388(17.1 \%)$ & \\
\hline Waist circumference & $82.6 \pm 8.8$ & $82.6 \pm 8.8$ & $82.5 \pm 8.7$ & 0.668 \\
\hline Triglyceride & $107.9 \pm 72.4$ & $107.1 \pm 72.1$ & $109.6 \pm 73.3$ & 0.172 \\
\hline High-density lipoprotein cholesterol & $53.9 \pm 12.0$ & $53.8 \pm 12.1$ & $53.9 \pm 12.0$ & 0.709 \\
\hline Fasting glucose & $98.2 \pm 16.6$ & $97.7 \pm 15.6$ & $99.5 \pm 18.5$ & 0.000 \\
\hline Systolic blood pressure & $115.4 \pm 13.2$ & $115.2 \pm 13.4$ & $115.7 \pm 12.9$ & 0.198 \\
\hline Diastolic blood pressure & $75.9 \pm 10.3$ & $75.6 \pm 10.4$ & $76.6 \pm 10.0$ & 0.000 \\
\hline Metabolic syndrome & $1320(18.2 \%)$ & $890(17.9 \%)$ & $430(19.0 \%)$ & 0.284 \\
\hline
\end{tabular}

A total of 7257 healthy individuals underwent regular health checkups, including white blood cell count, neutrophil, and lymphocyte counts. The mean age was $50.5 \pm 10.2$ years old, and $4208(58 \%)$ of the subjects were men. The mean NLR was $1.9 \pm 0.9$, and $10 \%$ of the population was in the increased NLR category. Metabolic syndrome was detected in $17.9 \%$ of the population. Based on previously described methods, 4986 subjects were classified into the discovery set, and 2270 were classified into the validation set. A quantile-quantile (Q-Q) plot is shown in Figure S2.

\subsection{Genome-Wide Association Study of the Neutrophil-Lymphocyte Ratio}

First, we carried out a GWAS in the discovery set with $P$-values below $5 \times 10^{-8}$ used as the threshold for significance after adjusting for age, sex, and BMI. In the discovery set, 9 SNPs were significantly associated with the log-transformed NLR. Among these 9 SNPs, 5 SNPs were imputed, and 4 SNPs were originally genotyped. The estimated imputation accuracies for the imputed SNPs 
were all greater than 0.9 , and their INFO values are represented in Table S1. We selected these SNPs for additional study in the validation set and found that all were significant ( $P$-values less than 0.05 , Table S1). Figure 1 shows the Manhattan plot of the GWAS of log-transformed NLR levels. Table S2 shows the linkage disequilibrium data for the significant SNPs (Figure S3). After these analyses, the SNPs were grouped into four regions: rs76596471, rs76181728, rs79945097 and rs7977554 near the TMEM116 gene, NAA25, and PTPN11 in chromosome 12; rs62065216, rs7502233, and rs7502539 near the THRA gene in chromosome 17; rs1879265 near the THRA gene in chromosome 17; and rs2102928 near the NR1D1 gene in chromosome 17.

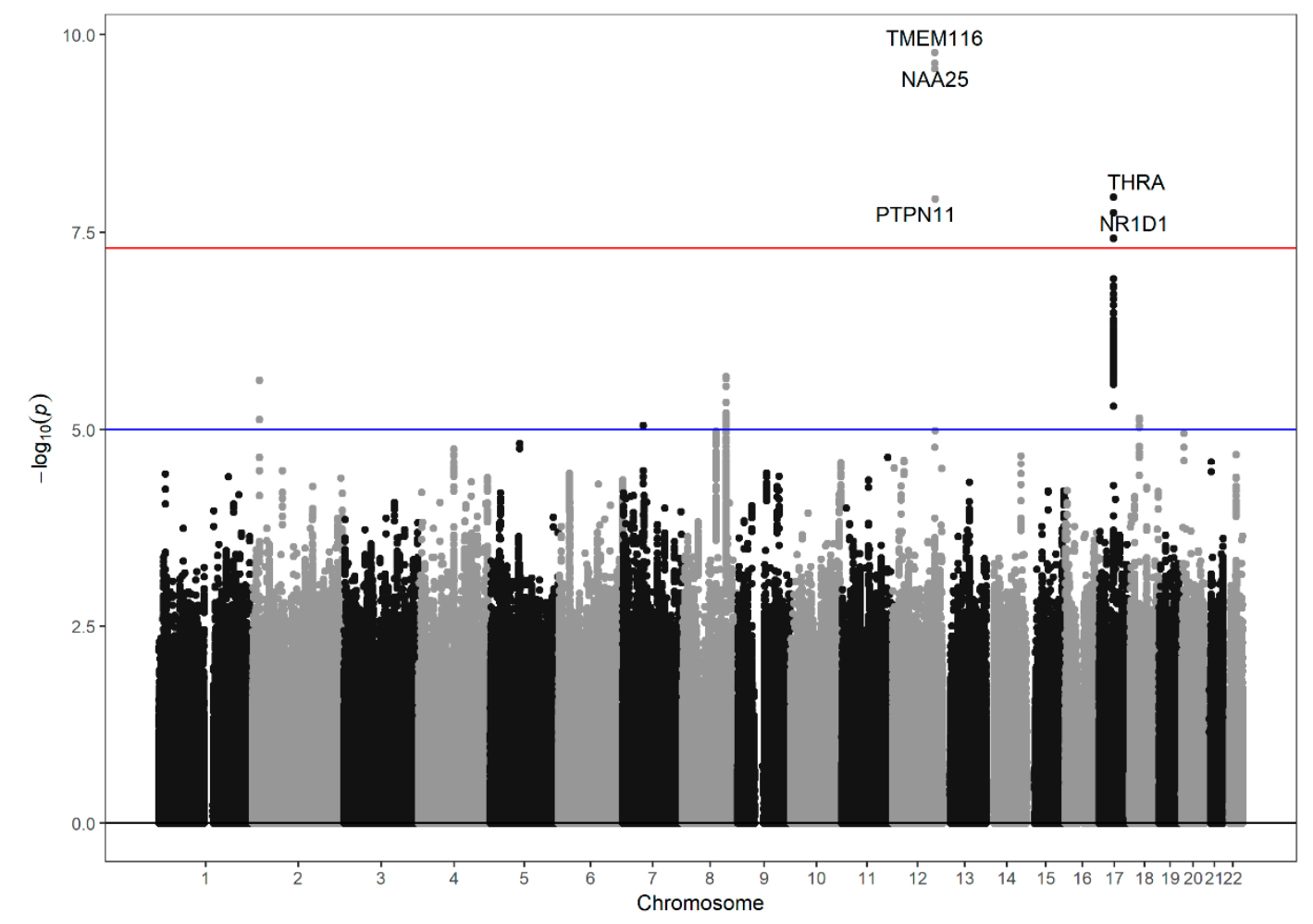

Figure 1. Manhattan plot of the strength of association $[-\log 10(P)$ values; $\mathrm{Y}$ axis] between single-nucleotide polymorphisms (SNPs) (X-axis by chromosome and chromosomal position) and log-transformed NLR levels. Threshold line: $5 \times 10^{-8}$.

Second, we performed the same GWAS for WBC counts, lymphocyte counts, and neutrophil counts because the NLR is a function of neutrophil and lymphocyte counts, both of which are included in the total WBC count. Hence, the association between NLR phenotype and significant SNPs might actually be related to the WBC, lymphocyte, or neutrophil status. The imputed SNPs rs76181728, rs76596471, rs7977554 and genotyped SNPs rs79945097 displayed a single-handed association with the NLR (Table S3). These four SNPs are located on chromosome 12 and were associated with the TMEM116, N-alpha-acetyltransferase 25, NatB auxiliary subunit (NAA25), and PTPN11 genes. Regarding linkage disequilibrium, all four SNPs had pairwise R2 values greater than 9.90 and D values greater than 0.98. A regional plot was obtained for the SNP rs76181728, which was most highly associated with the log-transformed NLR (Figure S4).

\subsection{Case-Control Study for the Presence of Metabolic Syndrome According to the 9 Significant SNPS}

We performed a case-control study using an additive model for each metabolic risk and metabolic syndrome with 9 SNPs that were significantly association with log-transformed NLRs when adjusted for age, sex, and BMI. Among the nine significant SNPs, three (rs76181728, rs79945097, and rs76596471) 
with a single-handed association with the NLR, as shown above, had a significant OR for increased blood pressure and increased waist circumference. Although these results did not reach statistical significance, the SNPs displayed a tendency toward an association with metabolic syndrome (Table 2).

Table 2. Case-control study to evaluate the associations among the significant SNPs and hypertension, increased waist circumference, and metabolic syndrome. OR: odds ratio; CI: confidence interval.

\begin{tabular}{|c|c|c|c|c|c|c|c|c|c|}
\hline \multirow[t]{2}{*}{ SNP } & \multicolumn{3}{|c|}{${ }^{a}$ Elevated Blood Pressure } & \multicolumn{3}{|c|}{$\begin{array}{l}\text { b Increased Waist } \\
\text { Circumference }\end{array}$} & \multicolumn{3}{|c|}{ Metabolic Syndrome } \\
\hline & OR & $95 \%$ CI & $P$-Value & OR & $95 \% \mathrm{CI}$ & $P$-Value & OR & $95 \%$ CI & $P$-Value \\
\hline rs76181728 & 1.348 & $1.111-1.635$ & 0.002 & 1.42 & $1.063-1.897$ & 0.017 & 1.253 & $0.972-1.615$ & 0.082 \\
\hline rs79945097 & 1.348 & $1.112-1.635$ & 0.002 & 1.424 & $1.067-1.901$ & 0.016 & 1.263 & $0.98-1.626$ & 0.071 \\
\hline rs76596471 & 1.341 & $1.105-1.627$ & 0.003 & 1.429 & $1.071-1.908$ & 0.015 & 1.266 & $0.983-1.631$ & 0.068 \\
\hline rs7977554 & 1.209 & $0.987-1.48$ & 0.067 & 1.44 & $1.071-1.935$ & 0.016 & 1.178 & $0.901-1.54$ & 0.231 \\
\hline rs7502539 & 1.072 & $0.974-1.181$ & 0.156 & 1.05 & $0.907-1.214$ & 0.515 & 1.09 & $0.96-1.237$ & 0.184 \\
\hline rs7502233 & 1.07 & $0.972-1.179$ & 0.167 & 1.057 & $0.914-1.222$ & 0.458 & 1.093 & $0.963-1.24$ & 0.168 \\
\hline rs1879265 & 1.046 & $0.95-1.15$ & 0.361 & 0.929 & $0.805-1.073$ & 0.318 & 0.89 & $0.784-1.009$ & 0.069 \\
\hline rs62065216 & 1.111 & $1.013-1.219$ & 0.025 & 0.984 & $0.856-1.131$ & 0.822 & 0.993 & $0.88-1.121$ & 0.912 \\
\hline rs2102928 & 1.007 & $0.878-1.155$ & 0.918 & 0.964 & $0.785-1.183$ & 0.724 & 0.956 & $0.799-1.143$ & 0.621 \\
\hline
\end{tabular}

Adjusted for age, sex, and BMI * Additive models were used for genotyping SNPs. ${ }^{\text {a }}$ Elevated blood pressure (blood pressure $\geq 130 / 85 \mathrm{mmHg}$ or use of medications for hypertension). ${ }^{\mathrm{b}}$ Increased waist circumference (males > $102 \mathrm{~cm}$; females $>88 \mathrm{~cm}$ ).

None of the SNPs was significantly associated with increased triglycerides, decreased HDL cholesterol or elevated fasting glucose (Table S4).

\subsection{Regression Models for Increased Blood Pressure, Decreased HDL Cholesterol and Metabolic Syndromes}

The top SNP associated with the log-transformed NLRs was rs76181728 in the TMEM116 gene $\left(P\right.$-value, discovery set $=1.68 \times 10^{-10}$; validation set $\left.=0.00749\right)$. We designed several regression models for increased blood pressure, decreased HDL cholesterol, and metabolic syndromes using various risk factors including rs76181728 as the dominant genotypic model (Table 3).

Table 3. Regression models for metabolic risks and metabolic syndrome with the SNP rs76181728 used as the dominant genotype.

\begin{tabular}{|c|c|c|c|c|}
\hline $\begin{array}{l}\text { Predicting } \\
\text { Feature }\end{array}$ & a Model 1 & b Model 2 & ${ }^{\mathrm{c}}$ Model 3 & ${ }^{d}$ Model 4 \\
\hline & $\begin{array}{c}\text { OR }(95 \% \mathrm{CI}) \\
P \text {-Value }\end{array}$ & $\begin{array}{l}\text { OR }(95 \% \mathrm{CI}) \\
P \text {-Value }\end{array}$ & $\begin{array}{l}\text { OR (95\% CI) } \\
P \text {-Value }\end{array}$ & $\begin{array}{l}\text { OR }(95 \% \mathrm{CI}) \\
P \text {-Value }\end{array}$ \\
\hline${ }^{\mathrm{e}}$ Increased NLR & $0.584(0.413-0.827), 0.002$ & $0.616(0.415-0.913), 0.015$ & & \\
\hline $\begin{array}{l}\text { Increased blood } \\
\text { pressure }\end{array}$ & $1.308(1.072-1.597), 0.008$ & 1.169 (0.928-1.474), 0.186 & $1.342(1.098-1.641), 0.004$ & $1.204(0.954-1.52), 0.118$ \\
\hline $\begin{array}{l}\text { Increased waist } \\
\text { circumference }\end{array}$ & 1.418 (1.054-1.908), 0.021 & 1.506 (1.075-2.11), 0.017 & 1.427 (1.059-1.924), 0.019 & 1.563 (1.114-2.193), 0.009 \\
\hline $\begin{array}{l}\text { Metabolic } \\
\text { syndrome }\end{array}$ & $1.242(0.957-1.612), 0.103$ & 1.418 (1.057-1.901), 0.019 & $1.27(0.977-1.651), 0.073$ & 1.465 (1.091-1.969), 0.011 \\
\hline \multicolumn{5}{|c|}{$\begin{array}{l}\text { a Model 1: adjusted for age, sex and BMI. }{ }^{\mathbf{b}} \text { Model 2: adjusted for age, sex, smoking status, alcohol consumption } \\
\text { and BMI. }{ }^{c} \text { Model 3: adjusted for age, sex, BMI and log-transformed NLR. }{ }^{\mathbf{d}} \text { Model } 4: \text { adjusted for age, sex, } \\
\text { smoking status, alcohol consumption, BMI, and log-transformed NLR. }{ }^{\mathbf{e}} \text { Normal NLR: NLR }<\text { mean NLR }+1 \\
\text { standard deviation vs. Increased NLR: NLR } \geq \text { mean NLR }+1 \text { standard deviation. Dominant models were used for } \\
\text { genotyping SNPs. For comparisons of the regression models, the reference was the model that used the AA or AG } \\
\text { allele (minor alleles), and the ORs were calculated by comparison with the GG allele (the major homologous allele). }\end{array}$} \\
\hline
\end{tabular}

The results showed that rs76181728 was significantly associated with increased blood pressure $(\mathrm{OR}=1.308,95 \% \mathrm{CI}=1.072-1.597, P=0.008)$ in model 1 (age-, sex- and BMI- adjusted). The SNP was also significantly associated $(\mathrm{OR}=1.342,95 \% \mathrm{CI}=1.098-1.641, P=0.004)$ after additional adjustment for the NLR phenotype (log-transformed NLR), as shown in model 3. All four models resulted in a significant OR for predicting increased waist circumference. Regression model 4 had the highest OR 
for increased waist circumference $(\mathrm{OR}=1.563,95 \% \mathrm{CI}=1.114-2.193, P=0.009)$ and was constructed using rs76181728 as the dominant genotype adjusted for age, sex, smoking status, alcohol consumption, BMI, and log-transformed NLR.

For metabolic syndrome, rs76181728 had a significant OR of 1.418 (95\% confidence interval $=1.057-1.901, P=0.019)$ in model 2 and showed a significant association $(\mathrm{OR}=1.465$, $95 \% \mathrm{CI}=1.091-1.969, P=0.011$ ) after additional adjustment for the NLR phenotype (log-transformed NLR) in model 4.

\section{Discussion}

This is the first GWAS to evaluate the NLR, and our results indicate that four novel SNPs, rs76181728, rs79945097, rs76596471 and rs7977554, are associated with the NLR. These markers are associated with three genes, PTPN11, NAA25, and TMEM116, that are located on chromosome 12. rs76181728, the most significant of the four SNPs, had the power to predict metabolic risks such as increased waist circumference, increased blood pressure and metabolic syndrome after adjustment for NLR phenotype (log-transformed NLR).

The NLR, which represents the balance between neutrophils and lymphocytes in the body, is a biomarker of systemic inflammation and has recently been the subject of substantial amount of research [15]. The NLR reflects processes in two different immune pathways: neutrophils reflect ongoing immune responses, while lymphocytes reflect immune regulatory pathways $[16,17]$. This balance between neutrophils and lymphocytes has been suggested to reflect the deep-seated immune status [1].

However, the pathophysiological processes that underlie disruptions to the balance between neutrophils and lymphocytes have not been identified, and the corresponding genetic background has not been reported. In this GWAS, nine SNPs were found to be associated with the NLR. As shown in Table S3, SNPs that were significantly associated with either neutrophil or lymphocyte counts were excluded so that only those SNPs that were significantly associated with the NLR were selected for analysis. Thus, only novel SNPs that are uniquely associated with NLR were identified.

We selected rs76181728, which had the highest significance among the identified novel SNPs, to construct a regression model for metabolic syndrome and metabolic risks. In future studies, further analyses could potentially use this marker to predict metabolic syndrome. We found that rs76181728 was independently associated with waist circumference, blood pressure and metabolic syndrome.

Interestingly, rs76181728 maintained its independent predictive power even when the models applied were adjusted for NLR phenotype (log-transformed NLR). Though the NLR phenotype is known to predict metabolic risks on its own, information related to this polymorphism may provide an additional effect for predicting metabolic risks. Therefore, SNPs associated with the NLR can be used as biomarker for metabolic syndrome or its associated risks, for which the NLR phenotype has previously been used as a biomarker. Even before the NLR phenotype manifests, SNPs associated with the NLR can be used as biomarkers to predict various diseases, such as metabolic syndrome and related risks. However, our findings should be replicated in other populations possessing different characteristics and applied to the analysis of cancer or cardiovascular disease regression models.

The novel SNPs identified in our study were associated with three genes, namely, PTPN11, NAA25, and TMEM116. The PTPN11 gene encodes protein tyrosine phosphatase non-receptor type 11 , also known as protein tyrosine phosphatase 1D, SHP-2 [18,19]. Protein tyrosine phosphatases are involved in signaling pathways that regulate cellular activation, proliferation and differentiation [20]. These signaling events are important for mitogenic activation, metabolic control, and cell migration [21, 22]. Evidence from a mouse model showed that SHP-2 regulates glucose and lipid metabolism [23], and in aged mice, hepatocyte-specific deletion of SHP-2 promoted inflammatory signaling and hepatic inflammation/necrosis [24]. Activating SHP-2 mutations have been observed in neuroblastoma, melanoma, breast cancer, lung cancer, and colorectal cancer [25]. Some GWASs have demonstrated 
an association between the PTPN11 gene and gastric cancer [26], colitis, and serum lipid levels [27,28]. Considering that there are pathophysiological associations between the PTPN11 gene and the diseases described above, PTPN11 could be involved in a mechanism that connects the NLR to inflammation, cancer and metabolic diseases.

The NAA25 gene encodes NAA25, which functions in normal cell cycle progression [29] and cell cycle regulation [30]. The TMEM116 gene encodes transmembrane protein 116, which has been reported to be associated with atrial fibrillation [31] and diabetes [32].

Linkage disequilibrium at the 12q24 locus has been hypothesized to interfere with genes such as CUTL2, FAM109A, SH2B3, ATXN2, BRAP, ACAD10, ALDH2, MAPKAPK5, TMEM116, ERP29 [9], NAA25/C12orf30, TRAFD1, HECTD4/C12orf51, RPL6, and PTPN11 [32-35], including the genes we identified by the nine novel SNPs. The 12q24 locus in humans has been suggested to be associated with susceptibilities to various diseases and conditions [32], such as hematopoietic diseases [36-38], type 1 diabetes [33,34,39,40], rheumatoid arthritis [41,42], abnormal serum urate levels [43], hypertension [35, 44], and obesity [45]. Given that all of the SNPs identified here were found to be associated with genes located at the 12q24 locus, the pathophysiology underlying and predictive function of the NLR in various diseases is likely to be associated with the status of the 12q24 locus. This phenomenon should be further investigated in biochemical studies and replicated in larger populations.

Our study has several limitations. First, we enrolled individuals undergoing self-paid comprehensive health checkups. Therefore, the socioeconomic characteristics of the populations was relatively good, and these individuals may be healthier than those in the average population. Second, we performed statistical analyses in discovery and validation sets but did not have a population for replication. Thus, these analyses should be repeated in a larger population with different characteristics. Third, all of the enrolled individuals were Korean. Our findings should therefore be replicated in populations with other ethnicities in future studies. Fourth, because the SNPs we discovered are novel, no previous papers have reported on their function. In future studies, experiments should be carried out to analyze the association between the novel SNPs and the protein expression of their related genes. Fifth, the clinical implications of the discovered SNPs should be evaluated in a larger variety of diseases, such as cancer and cardiovascular diseases. In this study, we included only metabolic syndrome in our analysis. It is our intention to collect more information in a broader variety of phenotypes in future studies. Sixth, blood parameters such as monocytes, lymphocyte-monocyte ratio, and neutrophil-monocyte ratio reflect the immune status. In this study, we did not collect the monocyte count during the data collection process. To elucidate the comprehensive effect of the genetic factors of myeloid compartments on various diseases, those parameters should be investigated further.

In conclusion, we identified novel SNPs that are associated with the NLR and showed that the most significant SNPs might be useful for predicting increased waist circumference, increased blood pressure, and metabolic syndrome even after adjustment for the NLR phenotype. Therefore, SNPs in the relevant genes at locus 12q24 might have a potential to be used as biomarkers for diseases that have previously been predicted only by the NLR phenotype. The predictive value of these SNPs as biomarkers should be further evaluated in various diseases, and the results should be replicated in other populations.

Supplementary Materials: The following are available online at http:/ /www.mdpi.com/2077-0383/7/8/204/s1, Table S1: SNPs associated with the log-transformed NLR, Table S2: Linkage disequilibrium among the discovered SNPs, Table S3: SNPs associated with log-transformed NLR, WBC count, lymphocyte count, and neutrophil count, Table S4: Case-control study of each metabolic risk according to the significant SNP, Figure S1: Principal component analysis (PCA) to adjust the population stratification, Figure S2: Q-Q plot of the log-transformed NLR genome-wide association study, Figure S3: Linkage disequilibrium plot among significant SNPs, Figure S4: Regional plot for the top SNP rs76181728 associated with the log-transformed NLR.

Author Contributions: Conceptualization, B.P. and E.K.C.; Methodology, B.P. and E.K.C.; Software, B.P. and S.W.; Validation, S.L., E.S. and H.Y.K.; Formal Analysis, B.P.; Investigation, S.L., E.S. and H.Y.K.; Resources, S.L., E.S. 
and H.Y.K.; Data Curation, E.K.C.; Writing-Original Draft Preparation, E.K.C.; Writing-Review \& Editing, S.L. and S.W.; Visualization, B.P.; Supervision, E.K.C.; Project Administration, E.K.C.; Funding Acquisition, E.K.C.

Funding: This research was funded by [Seoul National University Hospital Research Fund] Grant number [2620170070 (2017-3383)].

Acknowledgments: We express our special thanks to the members of DNA Link, Inc.

Conflicts of Interest: The authors declare no conflict of interest.

\section{References}

1. Paquissi, F.C. The predictive role of inflammatory biomarkers in atrial fibrillation as seen through neutrophil-lymphocyte ratio mirror. J. Biomark. 2016. [CrossRef] [PubMed]

2. Grenader, T.; Nash, S.; Adams, R. Derived neutrophil lymphocyte ratio is predictive of survival from intermittent therapy in advanced colorectal cancer: A post hoc analysis of the MRC COIN study. Br. J. Cancer 2016, 114, 612-615. [CrossRef] [PubMed]

3. Hu, K.; Lou, L.; Ye, J.; Zhang, S. Prognostic role of the neutrophil-lymphocyte ratio in renal cell carcinoma: A. meta-analysis. BMJ Open 2015, 5, e006404. [CrossRef] [PubMed]

4. Hu, Z.D.; Huang, Y.L.; Qin, B.D. Prognostic value of neutrophil to lymphocyte ratio for gastric cancer. Ann. Transl. Med. 2015, 3, 50. [PubMed]

5. Surendar, J.; Indulekha, K.; Mohan, V.; Pradeepa, R. Association of neutrophil-lymphocyte ratio with metabolic syndrome and its components in Asian Indians (CURES-143). J. Diabetes Complic. 2016, 30, 1525-1529. [CrossRef] [PubMed]

6. Suh, B.; Shin, D.W.; Kwon, H.M. Elevated neutrophil to lymphocyte ratio and ischemic stroke risk in generally healthy adults. PLOS ONE 2017, 12, e0183706. [CrossRef] [PubMed]

7. Chen, J.; Chen, M.H.; Li, S. Usefulness of the neutrophil-to-lymphocyte ratio in predicting the severity of coronary artery disease: A Gensini score assessment. J. Atheroscler. Thromb. 2014, 21, 1271-1282. [CrossRef] [PubMed]

8. Shah, N.; Parikh, V.; Patel, N. Neutrophil lymphocyte ratio significantly improves the Framingham risk score in prediction of coronary heart disease mortality: Insights from the National Health and Nutrition Examination Survey-III. Int. J. Cardiol. 2014, 171, 390-397. [CrossRef] [PubMed]

9. Lee, C.; Choe, E.K.; Choi, J.M. Health and Prevention Enhancement (H-PEACE): A. retrospective, population-based cohort study conducted at the Seoul National University Hospital Gangnam Center: Korea. BMJ Open 2018, 8, e019327. [CrossRef] [PubMed]

10. Grundy, S.M.; Brewer, H.B., Jr.; Cleeman, J.I. Definition of metabolic syndrome: Report of the National Heart, Lung, and Blood Institute/American Heart Association conference on scientific issues related to definition. Arterioscler Thromb. Vasc. Biol. Lung Nat. Heart 2004, 24, e13-e18. [CrossRef] [PubMed]

11. Choe, E.K.; Lee, Y.; Cho, J.Y. Search for genetic factor association with cancer-free prostate-specific antigen level elevation on the basis of a genome-wide association study in the Korean population. Eur. J. Cancer Prev. 2017, 27, 453-460. [CrossRef] [PubMed]

12. Delaneau, O.; Zagury, J.F.; Marchini, J. Improved Whole-chromosome phasing for disease and population genetic studies. Nat. Methods 2013, 10, 5-6. [CrossRef] [PubMed]

13. Howie, B.N.; Donnelly, P.; Marchini, J. A flexible and accurate genotype imputation method for the next generation of genome-wide association studies. PLoS Genet. 2009, 5, e1000529. [CrossRef] [PubMed]

14. Purcell, S.; Neale, B.; Todd-Brown, K. PLINK: A tool set for whole-genome association and population-based linkage analyses. Am. J. Hum. Genet. 2007, 81, 559-575. [CrossRef] [PubMed]

15. Liu, S.; Zheng, H.; Zhu, X. Neutrophil-to-lymphocyte ratio is associated with diabetic peripheral neuropathy in type 2 diabetes patients. Diabetes Res. Clin. Pract. 2017, 130, 90-97. [CrossRef] [PubMed]

16. Rajakariar, R.; Lawrence, T.; Bystrom, J. Novel biphasic role for lymphocytes revealed during resolving inflammation. Blood 2008, 111, 4184-4192. [CrossRef] [PubMed]

17. El Kebir, D.; Filep, J.G. Targeting neutrophil apoptosis for enhancing the resolution of inflammation. Cells 2013, 2, 330-348. [CrossRef] [PubMed]

18. Jamieson, C.R.; Van der Burgt, I.; Brady, A.F. Mapping a gene for Noonan syndrome to the long arm of chromosome 12. Nat. Genet. 1994, 8, 357-360. [CrossRef] [PubMed] 
19. Freeman, R.M.; Plutzky, J.; Neel, B.G. Identification of a human Src homology 2-containing protein-tyrosine-phosphatase: A putative homolog of Drosophila corkscrew. Proc. Natl. Acad. Sci. USA 1992, 89, 11239-11243. [CrossRef] [PubMed]

20. Pulido, R.; Krueger, N.X.; Serra-Pagès, C. Molecular characterization of the human transmembrane protein-tyrosine phosphatase delta. Evidence for tissue-specific expression of alternative human transmembrane protein-tyrosine phosphatase delta isoforms. J. Biol. Chem. 1995, 270, 6722-6728. [CrossRef] [PubMed]

21. Dechert, U.; Duncan, A.M.; Bastien, L. Protein-tyrosine phosphatase SH-PTP2 (PTPN11) is localized to 12q24.1-24.3. Hum Genet. 1995, 96, 609-615. [CrossRef] [PubMed]

22. Ahmad, S.; Banville, D.; Zhao, Z. A widely expressed human protein-tyrosine phosphatase containing Src homology 2 domains. Proc. Natl. Acad. Sci. USA 1993, 90, 2197-2201. [CrossRef] [PubMed]

23. Coulombe, G.; Rivard, N. New and unexpected biological functions for the Src-homology 2 domain-containing phosphatase SHP-2 in the gastrointestinal tract. Cell. Mol. Gastroenterol. Hepatol. 2016, 2, 11-21. [CrossRef] [PubMed]

24. Bard-Chapeau, E.A.; Li, S.; Ding, J. Ptpn11/Shp2 acts as a tumor suppressor in hepatocellular carcinogenesis. Cancer Cell 2011, 19, 629-639. [CrossRef] [PubMed]

25. Bentires-Alj, M.; Paez, J.G.; David, F.S. Activating mutations of the Noonan syndrome-associated SHP2/PTPN11 gene in human solid tumors and adult acute myelogenous leukemia. Cancer Res. 2004, 64, 8816-8820. [CrossRef] [PubMed]

26. Hishida, A.; Matsuo, K.; Goto, Y. Associations of a PTPN11 G/A polymorphism at intron 3 with Helicobactor pylori seropositivity, gastric atrophy and gastric cancer in Japanese. BMC Gastroenterol. 2009, 9, 51. [CrossRef] [PubMed]

27. Jamshidi, Y.; Gooljar, S.B.; Snieder, H. SHP-2 and PI3-kinase genes PTPN11 and PIK3R1 may influence serum apoB and LDL cholesterol levels in normal women. Atherosclerosis 2007, 194, e26-e33. [CrossRef] [PubMed]

28. Jia, Z.F.; Cao, X.Y.; Cao, D.H. Polymorphisms of PTPN11 gene could influence serum lipid levels in a sex-specific pattern. Lipids Health Dis. 2013, 12, 72. [CrossRef] [PubMed]

29. Starheim, K.K.; Arnesen, T.; Gromyko, D. Identification of the human N(alpha)-acetyltransferase complex B (hNatB): A complex important for cell-cycle progression. Biochem. J. 2008, 415, 325-331. [CrossRef] [PubMed]

30. Caesar, R.; Warringer, J.; Blomberg, A. Physiological importance and identification of novel targets for the N-terminal acetyltransferase NatB. Eukaryot. Cell 2006, 5, 368-378. [CrossRef] [PubMed]

31. Sinner, M.F.; Tucker, N.R.; Lunetta, K.L. Integrating genetic, transcriptional, and functional analyses to identify 5 novel genes for atrial fibrillation. Circulation 2014, 130, 1225-1235. [CrossRef] [PubMed]

32. Auburger, G.; Gispert, S.; Lahut, S. 12q24 locus association with type 1 diabetes: SH2B3 or ATXN2? World J. Diabetes 2014, 5, 316-327. [CrossRef] [PubMed]

33. Wellcome Trust Case Control Consortium. Genome-wide association study of 14,000 cases of seven common diseases and 3000 shared controls. Nature 2007, 447, 661-678. [CrossRef] [PubMed]

34. Cooper, J.D.; Walker, N.M.; Smyth, D.J. Follow-up of 1715 SNPs from the Wellcome Trust Case Control Consortium genome-wide association study in type I diabetes families. Genes Immun. 2009, 10, S85-SS94. [CrossRef] [PubMed]

35. Ikram, M.K.; Sim, X.; Jensen, R.A. Four novel Loci (19q13, 6q24, 12q24, and 5q14) influence the microcirculation in vivo. PLoS Genet. 2010, 6, e1001184. [CrossRef]

36. Takaki, S.; Sauer, K.; Iritani, B.M. Control of B cell production by the adaptor protein lnk. Definition of a conserved family of signal-modulating proteins. Immunity 2000, 13, 599-609. [CrossRef]

37. Bersenev, A.; Wu, C.; Balcerek, J. Lnk constrains myeloproliferative diseases in mice. J. Clin. Investig. 2010, 120, 2058-2069. [CrossRef] [PubMed]

38. Roberts, K.G.; Morin, R.D.; Zhang, J. Genetic alterations activating kinase and cytokine receptor signaling in high-risk acute lymphoblastic leukemia. Cancer Cell 2012, 22, 153-166. [CrossRef] [PubMed]

39. Todd, J.A.; Walker, N.M.; Cooper, J.D. Robust associations of four new chromosome regions from genome-wide analyses of type 1 diabetes. Nat. Genet. 2007, 39, 857-864. [CrossRef] [PubMed]

40. Cooper, J.D.; Walker, N.M.; Healy, B.C. Analysis of 55 autoimmune disease and type II diabetes loci: Further confirmation of chromosomes 4q27,12q13.2 and 12q24.13 as type I diabetes loci, and support for a new locus, 12q13.3-q14.1. Genes Immun. 2009, 10, S95-S120. [CrossRef] [PubMed] 
41. Hinks, A.; Martin, P.; Flynn, E. Investigation of type 1 diabetes and coeliac disease susceptibility loci for association with juvenile idiopathic arthritis. Ann. Rheum. Dis. 2010, 69, 2169-2172. [CrossRef] [PubMed]

42. Coenen, M.J.; Trynka, G.; Heskamp, S. Common and different genetic background for rheumatoid arthritis and coeliac disease. Hum. Mol. Genet. 2009, 18, 4195-4203. [CrossRef] [PubMed]

43. Köttgen, A.; Albrecht, E.; Teumer, A. Genome-wide association analyses identify 18 new loci associated with serum urate concentrations. Nat. Genet. 2013, 45, 145-154. [CrossRef] [PubMed]

44. Ehret, G.B.; Munroe, P.B.; International Consortium for Blood. Genetic variants in novel pathways influence blood pressure and cardiovascular disease risk. Nature 2011, 478, 103-109. [CrossRef] [PubMed]

45. Figueroa, K.P.; Farooqi, S.; Harrup, K. Genetic variance in the spinocerebellar ataxia type 2 (ATXN2) gene in children with severe early onset obesity. PLoS ONE 2009, 4, e8280. [CrossRef] [PubMed]

(C) 2018 by the authors. Licensee MDPI, Basel, Switzerland. This article is an open access article distributed under the terms and conditions of the Creative Commons Attribution (CC BY) license (http:/ / creativecommons.org/licenses/by/4.0/). 